\title{
LA INTELIGIBILIDAD DE ESTUDIANTES MEXICANOS AL PRONUNCIAR LAS CONSONANTES OCLUSIVAS SORDAS ASPIRADAS DEL INGLÉS
}

\section{THE INTELLIGIBILITY OF MEXICAN STUDENTS WHEN PRONOUNCING ENGLISH VOICELESS STOPS}

\author{
Milagros Eugenia Pérez Sámano \\ Mónica Sanaphre Villanueva² \\ Universidad Autónoma de Querétaro \\ México
}

\section{RESUMEN}

La articulación de los fonemas del inglés puede ser una tarea difícil para los estudiantes de esta

1 https://orcid.org/0000-0002-4733-541X milagros.e.samano@gmail.com, +5244212800 56 Universidad Autónoma de Querétaro. Milagros Eugenia Pérez Sámano es recién egresada del Doctorado en Lingüística por la Universidad Autónoma de Querétaro. Obtuvo su Maestría en Lingüística por la misma Universidad y tiene una Licenciatura en Lenguas Modernas- Inglés con línea terminal en Lingüística y Docencia. Su línea de especialidad es la adquisición y enseñanza de lengua.

2 https://orcid.org/0000-0003-4647-4378 sanaphre@gmail.com, +52 4423589024

Universidad Autónoma de Querétaro. Mónica Sanaphre Villanueva es profesora e investigadora del Centro de Estudios Literarios y Lingüísticos de la Facultad de Lenguas y Letras de la Universidad Autónoma de Querétaro su principal línea de interés es la adquisición del inglés como una segunda lengua o una lengua extranjera y el diseño efectivo de técnicas de enseñanza. lengua, ya que están obligados a diferenciar los fonemas que, por la influencia de la ortografía, asumen como equivalentes en inglés $y$ español. Estos desafíos fonéticos resultan en pronunciaciones inexactas causando problemas de inteligibilidad, lo que puede dificultar su comprensión al comunicarse con hablantes nativos. La inteligibilidad, definida como la precisión con la que un oyente comprende una expresión dada (Flege, 1988; Munro \& Derwing, 1995), depende de una gran variedad de factores atribuidos al hablante, al oyente y a la señal percibida. El objetivo de este estudio fue examinar el grado de inteligibilidad que presentan los fonemas oclusivos sordos $/ \mathrm{p}^{\mathrm{h}}, \mathrm{t}^{\mathrm{h}}$, $\mathrm{k}^{\mathrm{h}} /$ del inglés, en posición inicial de palabra, al ser pronunciadas por estudiantes mexicanos 
de inglés cuando son escuchadas por angloparlantes norteamericanos. Para lograrlo, creamos una prueba de inteligibilidad basada en el reconocimiento de palabras en la cual se le solicitó a los evaluadores norteamericanos que escucharan una palabra pronunciada por un estudiante mexicano y escribieran lo que creían haber escuchado. Los resultados mostraron que la ausencia de aspiración causa serios problemas de inteligibilidad en el fonema $/ \mathrm{p}^{\mathrm{h}} /$, y una afectación menos seria en la inteligibilidad de $/ \mathrm{t}^{\mathrm{h}} / \mathrm{y} / \mathrm{k}^{\mathrm{h}} /$, por lo que concluimos que los profesores tendrían que prestar mayor atención a la enseñanza de la pronunciación aspirada de $/ \mathrm{p}^{\mathrm{h}} /$.

PALABRAS CLAVE: inteligibilidad, estudiantes mexicanos, fonemas oclusivos sordos

\section{ABSTRACT}

One aspect of pronunciation that can be difficult for Mexican students of English is the articulation of phonemes since it is necessary to learn to differentiate phonemes that are perceived as corresponding due to their orthographical similarity. These phonetical challenges result in inaccurate pronunciation that may cause intelligibility problems and hinder communication with native speakers. Intelligibility, defined as the accuracy with which a listener understands a given utterance (Flege, 1988; Munro \& Derwing, 1995), depends on a wide variety of factors attributed to the speaker, the listener, and the perceived signal. The aim of this study was to measure how intelligible the pronunciation Mexican students made of the English voiceless, stop, aspirated consonants, $/ \mathrm{p}^{\mathrm{h}}, \mathrm{t}^{\mathrm{h}}, \mathrm{k}^{\mathrm{h}} /$, at the beginning of words resulted to 80 American English speakers. To achieve this, we created an intelligibility test based on word recognition in which American speakers were asked to listen to the word pronounced by a Mexican English student and write down what they thought they had heard. The results showed that the absence of aspiration produced by students caused severe intelligibility problems in the case of the phoneme $/ \mathrm{p}^{\mathrm{h}} /$, whereas phonemes $/ \mathrm{t}^{\mathrm{h}} /$ and / $\mathrm{k}^{\mathrm{h}} /$ resulted more intelligible. On this basis we conclude that English teachers should pay more attention to teaching the aspirated pronunciation of $/ \mathrm{p}^{\mathrm{h}} /$.

KEY WORDS: intelligibility, Mexican students, voiceless stop phonemes La inteligibilidad de estudiantes mexicanos al pronunciar las consonantes oclusivas sordas aspiradas del inglés.

\section{INTRODUCCIÓN}

Hoy en día, el inglés es la lengua más utilizada a nivel global para interactuar en sectores como la ciencia, la educación, la cultura, la salud, el comercio y el entretenimiento (Weil y Pullin, 2011). Es bien reconocido también (Acosta y Ovalle 2017) que existe una correlación positiva, directamente proporcional, entre el nivel de manejo de la lengua inglesa y el nivel educativo, la calidad de vida y los ingresos de las personas. Resulta, por tanto, de gran relevancia a nivel mundial, la búsqueda e implementación de las mejores metodologías, técnicas y materiales de enseñanza que nos permitan proporcionar a los estudiantes oportunidades para el óptimo desarrollo de las habilidades lingüísticas y la competencia comunicativa en la lengua inglesa.

Desafortunadamente, la tarea educativa no resulta fácil. Los profesores de inglés en Latinoamérica, y especialmente México, que es el contexto con el que estamos realmente familiarizadas, enfrentan una serie de carencias materiales y tecnológicas que imposibilitan el uso variado y abundante de materiales didácticos. Por otra parte, se trabaja usualmente con grupos numerosos en aulas pequeñas donde el trabajo en pares, tríos o grupos pequeños suele ser complicado y un gran consumidor del tiempo de la clase, por lo que los ejercicios 
de práctica oral e interacción suelen limitarse considerablemente. Además, en numerosas ocasiones los estudiantes viven en condiciones de pobreza que conllevan malnutrición, cansancio por el trabajo físico que realizan antes de asistir a la escuela, pocos recursos para adquirir libros y materiales, y poco tiempo para dedicarlo al estudio.

Entonces, al enfrentar todos estos retos en la enseñanza del inglés en México, nosotras, como profesoras, nos preguntamos: ¿en qué elementos debo centrar mi enseñanza para que los alumnos aprovechen al máximo el poco tiempo y los pocos recursos con los que cuentan para aprender inglés? Ante esta interrogante, hemos decidido trabajar en la identificación de algunos de los elementos centrales de la pronunciación segmental que puedan garantizar un manejo inteligible de la lengua inglesa.

Esta presentación es una parte de un proyecto mayor que tuvo como objetivo identificar los fonemas ingleses (consonantes líquidasróticas, fricativas, oclusivas sordas, vocales y diptongos) que, al ser pronunciados por nuestros estudiantes mexicanos, causaban problemas de inteligibilidad en los hablantes de inglés norteamericano. Al identificar esos fonemas, podremos orientar a otros profesores para que, al trabajar los fonemas específicos que causan problemas de inteligibilidad, garanticen que sus estudiantes serán comprendidos al interactuar con otros hablantes, independientemente del bajo desarrollo de su habilidad para articular todos fonemas ingleses o del acento latino que pudieran mostrar al hablar inglés. Como otros investigadores han mostrado (Duff, 2012), el acento -en nuestro caso, latino- al hablar otra lengua es una muestra de identidad que no deseamos eliminar; lo que buscamos garantizar es un alto nivel de inteligibilidad sin necesariamente erradicar el acento que el estudiante pueda tener.
Paralograresteobjetivoserealizarongrabaciones de estudiantes mexicanos leyendo en voz alta una serie de palabras que ellos conocían bien y que incluían todos los fonemas bajo estudio. Con el propósito de identificar los rasgos típicos del inglés que los estudiantes mexicanos no lograban pronunciar de manera cercana, tales grabaciones fueron analizadas acústicamente de manera detallada y contrastadas con los parámetros acústicos típicos de los hablantes norteamericanos. Enseguida, se pidió a 80 angloparlantes norteamericanos, residentes en diferentes estados de los Estados Unidos, que escucharan esas palabras y escribieran lo que habían escuchado. Sus respuestas nos permitieron identificar, por una parte, los fonemas que son identificados correctamente y no causan problemas de inteligibilidad, y por otra, los fonemas que causaron problemas e impidieron la correcta identificación de la palabra pronunciada por el estudiante mexicano.

En este artículo mostraremos los resultados que obtuvimos de la evaluación de los fonemas sordos aspirados del inglés $\left(/ \mathrm{p}^{\mathrm{h}} /, / \mathrm{t}^{\mathrm{h}} /, / \mathrm{k}^{\mathrm{h}} /\right)$. Como detallaremos más adelante, el rasgo de aspiración, típico del inglés, no es un rasgo significativo en español, por lo que los estudiantes mexicanos tienden a no pronunciarlo. Los resultados mostraron que la ausencia de este rasgo causa problemas serios de inteligibilidad en el fonema $/ \mathrm{p}^{\mathrm{h}} /$, pero no afecta de manera seria la inteligibilidad de $/ t^{h /} / \mathrm{y}^{\mathrm{h}} /$, por lo que los profesores tendrían que prestar mayor atención a la enseñanza de la pronunciación aspirada de $/ \mathrm{p}^{\mathrm{h}} /$.

El orden que seguiremos en esta presentación es el siguiente: Primero, haremos un recuento breve de las características acústicas de las consonantes aspiradas sordas inglesas / $\mathrm{p}^{\mathrm{h}} /, \mathrm{t} \mathrm{th}, \mathrm{k} / \mathrm{k}^{\mathrm{h}} /$. Enseguida, describiremos la metodología detallada del estudio, incluyendo sus participantes, la prueba de inteligibilidad y el análisis de los resultados. En la sección 
4, mostraremos los resultados obtenidos, y, finalmente, en la sección 5 , presentaremos algunas conclusiones y propuestas para implementar en el salón de clases.

\section{CARACTERÍSTICAS FONÉTICAS DE} $/ \mathrm{p}^{\mathrm{h}} /, / \mathrm{t}^{\mathrm{h}} /, / \mathrm{k}^{\mathrm{h}} /$

El español y el inglés son lenguas que utilizan el mismo alfabeto. Sin embargo, aunque utilizamos las mismas grafías al escribir, éstas no corresponden a los mismos sonidos o fonemas que producimos al hablar. La realización de los fonemas es diferente articulatoriamente en cada lengua, lo que resulta en diferentes características acústicas también.

Las consonantes $\mathrm{p}, \mathrm{t}, \mathrm{k}$, conocidas como oclusivas sordas en ambas lenguas, se diferencian porque, en inglés, presentan un rasgo específico en su pronunciación conocido como aspiración. La aspiración se define como una explosión de aire que se libera tras la oclusión de la consonante y se representa con una pequeña h super indexada al fonema, así: / $\mathrm{p}^{\mathrm{h}} /, / \mathrm{t}^{\mathrm{h}} /, / \mathrm{k}^{\mathrm{h}} /$. Este rasgo, ausente en el español, se realiza, en inglés, cuando las oclusivas sordas se ubican en posición inicial de palabra.

Una manera de medir el grado de aspiración que presenta una consonante es obteniendo el llamado VOT (Voice Onset Time), por sus siglas en inglés, que corresponde al tiempo, medido en milisegundos, que va del punto de cierre de la consonante hasta el inicio de la vocal que sigue a la consonante (Roach, 2009). Según autores como Toribio, et al. (2005), que proporcionan rangos obtenidos después de medir las consonantes en una amplia cantidad de hablantes nativos del inglés, la aspiración de $/ \mathrm{p}^{\mathrm{h}} /$ que realiza un angloparlante es de 55 milisegundos, la aspiración de $/ \mathrm{t}^{\mathrm{h}} /$ es de 80 milisegundos y la aspiración de $/ \mathrm{k}^{\mathrm{h}} /$ es de 70 milisegundos. Tomaremos estos rangos como un punto de referencia para evaluar el grado de aspiración que producen los estudiantes mexicanos al pronunciar estos fonemas en inglés.

Por otra parte, de acuerdo con Flege (1988), los hablantes de español realizamos una aspiración mínima de 26 milisegundos aproximadamente al articular las oclusivas sordas. Podemos esperar, entonces, que los estudiantes mexicanos produzcan las consonantes sordas inglesas con el grado de aspiración que realizan típicamente al hablar español. Pues, Barlow, et al. (2013) han demostrado ya que los aprendices de segundas lenguas tienden a transferir las características de la pronunciación de su lengua materna a la segunda lengua.

Siguiendo estos datos, podemos hipotetizar que nuestros estudiantes mexicanos de inglés presentarán diferencias notorias en la pronunciación de las consonantes oclusivas sordas inglesas al transferir la pronunciación del español al inglés. De aquí, la pregunta que buscamos responder es: ¿la diferencia en aspiración producida por los estudiantes mexicanos causa problemas de inteligibilidad cuando son escuchados por hablantes norteamericanos? ¿Resultan los tres fonemas igualmente ininteligibles?

Pasemos ahora a la descripción detallada de la metodología que seguimos.

\section{METODOLOGÍA}

\section{a. Participantes mexicanos}

Para este estudio participaron voluntariamente 12 estudiantes de inglés ( 6 hombres y 6 mujeres) de edades entre los 25 y 50 años (promedio de 38.33). Todos ellos residentes de la ciudad de Querétaro. Se les aplicó la prueba TOEFL con la cual pudimos determinar su nivel de dominio del inglés. La mitad del grupo ( 3 h y $3 \mathrm{~m}$ ) obtuvieron un nivel avanzado; y la otra mitad, (3 h y $3 \mathrm{~m}$ ), obtuvieron un nivel intermedio. 
Los 6 hombres de este estudio reportaron que su edad de inicio del aprendizaje del inglés fue durante su adolescencia, a partir de los 12 años de edad; al contrario de las mujeres, quienes reportaron que su edad de inicio de aprendizaje fue a los 6 años de edad. Los estudiantes reportaron haber viajado a un país de habla inglesa con un tiempo de permanencia que variaba de entre 5 días a 1 mes de estancia.

Todo el grupo reportó haber tenido clases de inglés con maestros mexicanos, en las cuales no se les permitía interactuar en español, pero no se asignaba tampoco tiempo para la enseñanza y la práctica específica de la pronunciación. Todo el grupo indicó estar motivado para aprender inglés ya que consideran que, si logran comunicarse efectivamente en dicha lengua, obtendrán mejores oportunidades laborales.

\section{b. Material de grabación}

Los participantes de este estudio fueron grabados mientras leían una lista que incluía 15 palabras que iniciaban con la consonante oclusiva sorda aspirada. En la Tabla 1, puede revisarse esta lista.

Las palabras fueron seleccionadas de acuerdo con los siguientes criterios: (1) cada una de las palabras estaba conformada por una sola sílaba; (2) todas eran palabras de contenido léxico, es decir, sustantivos o verbos; (3) todas las palabras eran de alta frecuencia de acuerdo con los parámetros establecidos por Leech, et al. (2001); (4) todas las palabras eran conocidas por los estudiantes mexicanos que las leyeron; (5) la consonante aspirada sorda se encontraba en posición inicial de palabra.

Tabla 1. Palabras pronunciadas y grabadas por los estudiantes nativo hablantes del español.

\begin{tabular}{|c|c|c|}
\hline$/ \mathbf{p}^{\mathrm{h}} /$ & $/ \mathrm{t}^{\mathrm{h}} /$ & $/ \mathbf{k}^{\mathrm{h}} /$ \\
\hline pay & tear & cap \\
pie & tie & came \\
pig & tip & cave \\
pin & town & cold \\
pill & two & kill \\
\hline
\end{tabular}

\section{c. Evaluadores norteamericanos}

Un grupo de 82 adultos, voluntarios, nativo hablantes del inglés americano, residentes en diferentes estados de Los Estados Unidos, fungieron como evaluadores de nuestra prueba en modalidad online. Estos evaluadores (70 mujeres, 12 hombres) se encontraban viviendo en diferentes estados al momento de la evaluación: 64 de ellos se encontraban en Wisconsin, 6 en California, 2 en Tennessee, 2 en Florida, 1 en Texas, 1 en Rhode Island, 1 en Oregón, 1 en Ohio, 1 en Carolina del Norte, 1 en Nueva York, 1 en Illinois y 1 en Georgia.

Las edades de los participantes van de los 18 a los 70 años (edad promedio $=25$ años). Ninguno reportó tener algún problema auditivo o de lenguaje. Su dialecto se determinó a partir de un mapa de dialectos de Estados Unidos en el que cada uno de los participantes indicó cuál de los acentos regionales, delimitados en el mapa, mejor describía su propio dialecto: $85.4 \%(n=70)$ del grupo indicó que su dialecto pertenecía al Norte Alto, $9.8 \%(n=8)$ indicó que su dialecto pertenecía al Sureste, $3.7 \%(n=3=)$ indicó que su dialecto pertenecía al Norte Bajo y $1.2 \%(n=1)$ indicó que su dialecto pertenecía al Sur Bajo.

Los participantes también registraron su exposición diaria a un acento extranjero en una escala del 1 al 5 , en la cual $1=$ nada de exposición o muy breve exposición y 5= exposición diaria. El 52.4\%(n=43) del grupo reportó que casi nunca (2) escucha un acento extranjero, el $26.8 \%(n=22)$ del grupo reportó que 
la mayor parte del tiempo (4) estaba expuesto a un acento extranjero, y el $20.7 \%(n=17)$ restante reportó tener una exposición diaria (5) a un acento extranjero.

\section{d. La prueba de inteligibilidad}

La prueba está compuesta por dos partes. La primera, conformada por un total de 16 preguntas, busca obtener el perfil lingüístico de los evaluadores (edad, dialecto, ocupación, contacto con acentos extranjeros). La segunda parte está constituida por un total de 30 audios de las palabras descritas anteriormente (véase Tabla 1.), leídas en voz alta por estudiantes mexicanos de inglés (Cfr. sección 3. a. Participantes mexicanos).

Durante la prueba, los evaluadores norteamericanos escuchaban cada audio y, a continuación, escribían lo que habían entendido. Esta prueba les tomaba alrededor de 30 minutos. Ninguno de los participantes expresó haber tenido dificultades para realizar la prueba.

\section{e. Análisis de resultados de la prueba}

Una vez que se obtuvieron todas las respuestas de cada evaluador, se llevó a cabo el siguiente análisis: se otorgaba 1 punto, si la consonante inicial había sido identificada correctamente por el evaluador. Se asignaba un 0 si la consonante no había sido identificada.

Se consideró que la consonante había sido identificada si (1) se reconocía la palabra correctamente, o (2) aunque no se reconocía la palabra correctamente, la opción proporcionada por el evaluador comenzaba con el mismo fonema en cuestión.

Finalmente, los errores producidos por los evaluadores se analizaron para identificar otras posibles causas de ininteligibilidad.

\section{RESULTADOS}

El análisis de las 82 evaluaciones recolectadas dio un total de 2,460 palabras a analizar (820 por fonema $/ \mathrm{p}^{\mathrm{h}}, \mathrm{t}^{\mathrm{h}}, \mathrm{k}^{\mathrm{h}} /$ ). En términos generales, obtuvimos que $/ \mathrm{p}^{\mathrm{h}} /$ fue el fonema que más conflicto causó en la comprensión de los nativo hablantes, pues únicamente el 15\% de las veces se identificó correctamente (122 de 820 ocasiones). El fonema $/ \mathrm{t}^{\mathrm{t}} / \mathrm{se}$ evaluó correctamente el $49.5 \%$ de las veces (406 de 820 evaluaciones). Y, por último, el fonema $/ \mathrm{k}^{\mathrm{h}}$ / se evaluó correctamente el $58.5 \%$ de las veces (480 de 820 evaluaciones), siendo el que menor ininteligibilidad causó. La Figura 1 muestra estos resultados.

Figura 1. Comprensión de los evaluadores norteamericanos por cada consonante

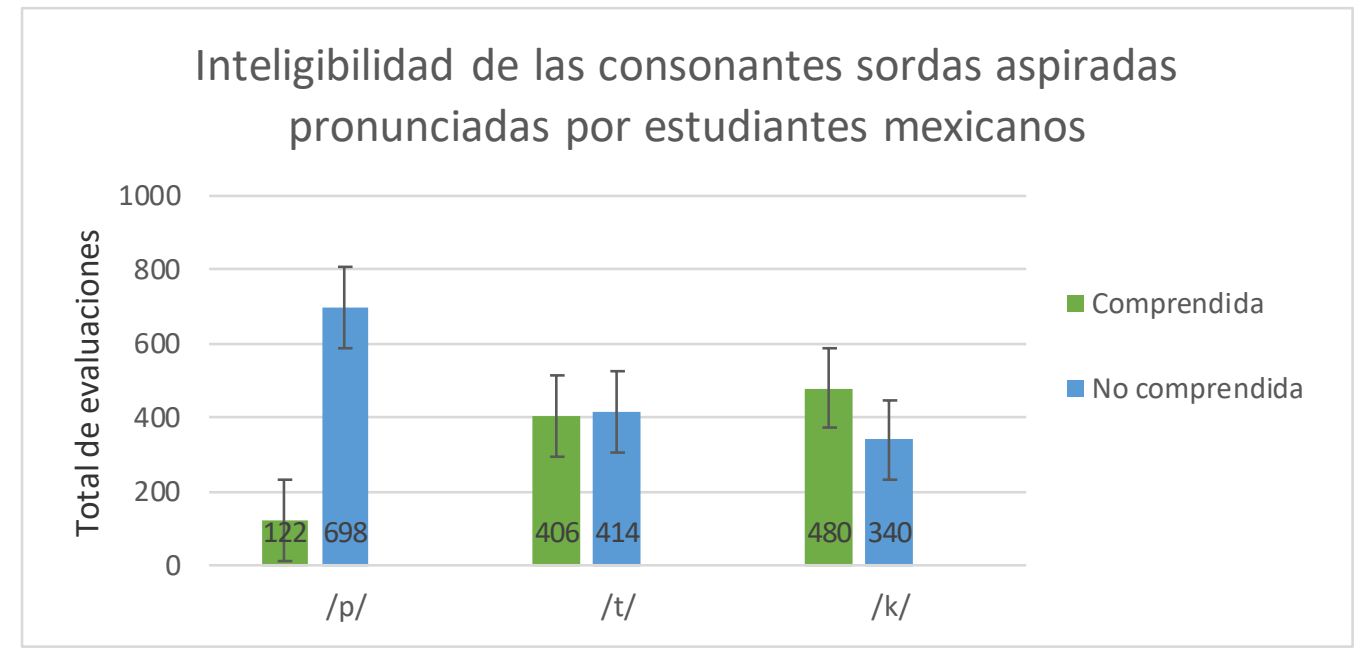


Se continuó con un análisis estadístico por medio de un ANOVA de una vía para corroborar la relevancia de la información obtenida, detectando un diferencia estadísticamente significativa entre las tres consonantes $[F(2,27)=$ $7.351, p=0.003]$. Es decir que, a pesar de que los tres fonemas carecen del rasgo de aspiración en la pronunciación de los estudiantes mexicanos, esta carencia no causa las mismas dificultades de comprensión en los interlocutores nativo hablantes del inglés, y es el fonema $/ \mathrm{p}^{\mathrm{h}} /$ el que más ininteligibilidad causó.

Esto nos indica que, como maestros de inglés, dentro de este grupo de consonantes, debemos guiar a nuestros alumnos para que centren su atención en realizar una pronunciación más clara y mejor articulada del fonema $/ \mathrm{p}^{\mathrm{h}} /$, ya que, si no se pronuncia con suficiente aspiración, causa mayores problemas en la comprensión de los nativo hablantes.
Posteriormente, utilizando el programa de análisis fonético PRAAT, (Boersma y Weenink (2021)) analizamos acústicamente la duración de la aspiración en la producción de los alumnos mexicanos para describir por qué su pronunciación mostraba problemas en la comprensión de los nativo hablantes.

La siguiente tabla muestra la información obtenida a partir del análisis. En la primera fila recordamos los parámetros de aspiración que produce el angloparlante al pronunciar palabras con cada uno de los fonemas analizados. La segunda fila indica los rangos en milisegundos en las grabaciones de los estudiantes mexicanos cuando el fonema no fue comprendido por los evaluadores norteamericanos $\mathrm{y}$, por último, indicamos el rango de aspiración en milisegundos en las grabaciones en las que el fonema sí fue reconocido correctamente por los nativo hablantes.

Tabla 2. Rangos de aspiración inteligibles y no inteligibles

\begin{tabular}{|c|c|c|c|}
\cline { 2 - 4 } \multicolumn{1}{c|}{} & $/ \mathbf{p}^{\mathrm{h}} l$ & $/ \mathbf{t}^{\mathrm{h}} /$ & \\
\hline $\begin{array}{c}\text { Aspiración típica del } \\
\text { angloparlante } \\
\text { (Toribio, et al. 2005) }\end{array}$ & $\begin{array}{c}55 \text { milisegundos } \\
(\mathrm{ms})\end{array}$ & $80 \mathrm{~ms}$ & $70 \mathrm{~ms}$ \\
\hline $\begin{array}{c}\text { Rango de aspiración que } \\
\text { no fue inteligible para los } \\
\text { evaluadores norteamericanos }\end{array}$ & entre $27-35 \mathrm{~ms}$ & entre $41-49 \mathrm{~ms}$ & entre $50-55 \mathrm{~ms}$ \\
\hline $\begin{array}{c}\text { Rango de aspiración que } \\
\text { sí fue inteligible para los } \\
\text { evaluadores norteamericanos }\end{array}$ & entre $36-44 \mathrm{~ms}$ & entre $50-64 \mathrm{~ms}$ & entre $56-60 \mathrm{~ms}$ \\
\hline
\end{tabular}

Estos resultados indican que, si nuestros alumnos no logran acercarse a los 40 milisegundos aproximadamente al pronunciar la aspiración de la $/ \mathrm{p}^{\mathrm{h}} /$ tendrán problemas para ser comprendidos por los nativo hablantes. En cuanto a $/ \mathrm{t}^{\mathrm{h}} /$ nuestros alumnos deberán lograr pasar de los 50 milisegundos de aspiración en promedio para alcanzar un rango esperado por los nativo hablantes, ya que ellos pronuncian esta consonante en unos 80 milisegundos de aspiración. Por último, nuestros alumnos deben pasar los 55 milisegundos de aspiración al pronunciar $/ \mathrm{k}^{\mathrm{h}} /$ para ser comprensibles.

Manejar una aspiración corta al transferir la pronunciación del español al inglés, tiene efectos tangibles en la percepción y comprensión de los nativo hablantes. Esto lo pudimos observar a partir de las respuestas de los evaluadores a cada uno de los audios que no fue comprendido. El análisis de las palabras no reconocidas por los evaluadores mostró que ellos escuchaban 
la contraparte sonora $/ \mathrm{b} /$, (en lugar de $/ \mathrm{p}^{\mathrm{h}} /$ ), en $68 \%$ de las ocasiones; /d/ (en lugar de $/ \mathrm{t}^{\mathrm{h}} /$ ) el $38 \%$ de las veces, $\mathrm{y} / \mathrm{g} /$ (en lugar de $/ \mathrm{k}^{\mathrm{h}} /$ ) un $35 \%$, cuando la aspiración no se articulaba dentro de los rangos de aspiración adecuados. En la siguiente tabla (Tabla 3.) podemos ver, en la segunda columna, la mayoría de las respuestas que dieron los evaluadores para cada palabra no inteligible.

Tabla 3. Respuestas de los evaluadores cuando el fonema no fue comprendido.

\begin{tabular}{|c|c|c|}
\hline $\begin{array}{c}\text { Palabra pronunciada por } \\
\text { los estudiantes mexicanos }\end{array}$ & $\begin{array}{c}\text { Palabra percibida } \\
\text { por los evaluadores } \\
\text { norteamericanos }\end{array}$ & $\begin{array}{c}\text { Porcentaje del total de las } \\
\text { respuestas analizadas por } \\
\text { fonema }\end{array}$ \\
\hline pay & bye & \\
pie & big & $68 \%$ \\
pig & bill & \\
pill & bin & \\
pin & dear & \\
tear & die & $38 \%$ \\
tie & dip & \\
tip & down & \\
town & do & \\
two & game & \\
came & gave & \\
cave & gap & \\
cap & gold & \\
cold & gill & \\
kill & & \\
\hline
\end{tabular}

Aunque las respuestas, como se puede apreciar en la Tabla 3, en su mayoría corresponden a consonantes oclusivas sonoras que son la contraparte de las oclusivas sordas, se obtuvieron también otras respuestas (Tabla 4) que distan significativamente de lo esperado. Las palabras que se presentan en la segunda columna de la Tabla 4 indican que, a pesar de no comprender el fonema, los evaluadores hacían un intento por categorizar, dentro de su repertorio fonético, lo que habían escuchado. Obsérvese cómo la falta de la aspiración llevó a los evaluadores a "escuchar" palabras completamente alejadas de lo que el estudiante mexicano intentaba producir, dejando clara la importancia que la pronunciación del rasgo de aspiración tiene en la inteligibilidad y comunicación exitosa del estudiante.

Tabla 4. Otras respuestas de los evaluadores cuando el fonema no fue comprendido.

\begin{tabular}{|c|c|c|}
\hline $\begin{array}{c}\text { Palabra pronunciada por los } \\
\text { estudiantes mexicanos }\end{array}$ & $\begin{array}{c}\text { Palabra percibida } \\
\text { por los evaluadores } \\
\text { norteamericanos }\end{array}$ & $\begin{array}{c}\text { Porcentaje del total de las } \\
\text { respuestas por fonema }\end{array}$ \\
\hline pay & $\begin{array}{c}\text { egg, they, very } \\
\text { l, eye, fine, find, hi }\end{array}$ & \\
pie & -- & $32 \%$ \\
pig & ill, feel, fill, hill & \\
pill & think, thin, theme & \\
pin & & \\
\hline
\end{tabular}




\begin{tabular}{|c|c|c|}
\hline tear & $\begin{array}{c}\text { clear, near } \\
\text { thigh, pie, why } \\
\text { tie }\end{array}$ & $62 \%$ \\
tip & feet, thin, pill, pink & \\
town & thief, teeth, grief & \\
two & thing, pink, cling & \\
\hline came & stop, pick up & \\
cave & hold & \\
cap & heal, little, middle & \\
cold & kill & \\
\hline
\end{tabular}

\section{DISCUSIÓN Y CONCLUSIONES}

Los resultados de este estudio nos permiten concluir que los estudiantes mexicanos tienden a producir las consonantes oclusivas sordas del inglés sin articular el rasgo de aspiración característico de tales consonantes inglesas. La falta de aspiración causa problemas de inteligibilidad severos ( $85 \%$ de ininteligibilidad) para el fonema $/ \mathrm{p}^{\mathrm{h}} /$, que tiende a comprenderse como su contraparte sonora no aspirada /b/. Los fonemas /t/ y /k/ al no presentar aspiración causaron problemas de inteligibilidad el $50 \%$ y el $59 \%$ de las veces que se pronunciaron. En el caso de estos dos fonemas, los evaluadores norteamericanos tendían a comprenderlos en su mayoría como otros fonemas no relacionados.

La tendencia que presentan los estudiantes mexicanos de realizar una muy breve o nula aspiración responde, en primer lugar, a los procesos típicos de transferencia de la lengua materna presentes en los procesos de adquisición-aprendizaje de lenguas extranjeras (Barlow, et al. 2013), a partir de los cuales, los estudiantes transfieren la pronunciación que conocen de las oclusivas sordas del español mexicano a las oclusivas sordas aspiradas del inglés.

En segundo lugar, la ausencia de la aspiración responde también al hecho, documentado por Piske, et al. (2001), de que los aprendices escuchan el inglés a partir de su lengua materna, el español, y dado que el rasgo de aspiración no se encuentra presente en su repertorio fonético español, no lo notan cuando lo escuchan en inglés.

En tercer lugar, los estudiantes mexicanos también son influidos por la similitud ortográfica inglés-español al representar los dos grupos de consonantes -oclusivas sordas aspiradas y oclusivas sordas no aspiradas- con las mismas grafías $p, t, k$. Como Bassetti y Atkinson (2015) han demostrado, la ortografía influencia significativamente la pronunciación que hacen los aprendices de las lenguas extranjeras.

A partir de estas observaciones, proponemos las siguientes estrategias que pueden implementar los docentes de inglés dentro de los ambientes educativos:

1. Guiar la atención de los alumnos, a través de comparaciones y explicaciones, para hacerlos notar y ser conscientes de que las consonantes del español y el inglés $-p, t, k$-, no se pronuncian igual en ambas lenguas a pesar de que se representen con las mismas grafías.

2. Aprovechar el uso constante que los alumnos hacen de sus dispositivos móviles para que, a través de grabaciones breves de sus propias voces, puedan monitorear su pronunciación, contrastarla con la pronunciación nativa y hacer los ajustes pertinentes en su articulación. De esta manera, podemos desarrollar en ellos la consciencia de que ellos mismos, de 
manera autodidacta, pueden modificar y mejorar su pronunciación fácilmente. Esto elevará su confianza y seguridad al hablar inglés, pues, por una parte, notarán que pronunciar mejor no es tan difícil como creían y, por otra, tendrán la certeza de que serán comprendidos exitosamente por otros hablantes internacionales.

3. Como docentes de inglés, debemos evitar ser la única fuente de modelos de pronunciación de la lengua inglesa de nuestros alumnos. Es importante familiarizarlos con diferentes materiales audiovisuales, aplicaciones, juegos, música -accesibles en sus dispositivos electrónicos, computadoras y televisores en casa- en los que la pronunciación nativa se escucha frecuentemente $y$ orientarlos para que observen el uso de la lengua o la pronunciación en diferentes contextos y puedan reproducirla.

4. Hacer uso de listas de palabras que contengan un fonema específico para corregir aspectos de su pronunciación mientras se leen en voz alta, tanto de manera individual como grupal. Es importante resaltar que, aun cuando en la vida cotidiana y laboral, la comunicación se da en contexto y no en forma de palabras aisladas, cuando se trata de trabajar pronunciación, las listas de palabras nos ofrecen la oportunidad de dirigir la atención de los estudiantes a detalles específicos que se tienen que trabajar, evitando las distracciones que otras palabras en oraciones y frases pudieran causar.

5. Hacer uso recurrente de videos y dirigir la atención de nuestros alumnos para que observen las gesticulaciones $y$ movimientos faciales que acompañan la articulación de los fonemas, pues como Sanaphre y Castellanos (2018) han mostrado, la observación de los gestos faciales facilita el aprendizaje de la pronunciación.

6. Por último, queremos hacer hincapié en que mientras nuestros alumnos se mantengan altamente motivados, su aprendizaje del inglés como lengua extranjera, y por ende su pronunciación, avanzará exitosamente. Por tanto, es necesario que hagamos hincapié constantemente en los beneficios educativos, profesionales, en mejora de la calidad de vida y hasta de entretenimiento que conlleva el aprendizaje de lenguas extranjeras.

\section{REFERENCIAS BIBLIOGRÁFICAS}

Acosta, N. K. M., y Ovalle, T. D. C. T. (2017). Estrategia para disminuir el índice de reprobación en la unidad de aprendizaje de inglés en una institución de nivel medio superior. Revista Electrónica del Desarrollo Humano para la Innovación. vol. 4, núm. 8., p.p. 1-14.

Bassetti, B. y Atkinson, N. (2015) "Effects of orthographic forms on pronunciation in experienced instructed second language learners". Applied Pshycolinguistics. vol.36, núm.1, p.p. 67-91.

Barlow, J. A., Branson, P. E., y Nip, I. S. (2013). Phonetic equivalence in the acquisition of//by Spanish-English bilingual children. Bilingualism: Language and Cognition, vol.16, núm. 1, p.p.68-85.

Boersma, Paul \& Weenink, David (2021). Praat: doing phonetics by computer [Computer program]. Version 6.1.51, retrieved 22 July 2021 from http://www.praat.org/ 
Duff, P. (2012). "Identity, agency, and second language acquisition" en Gass, S. y Mackey, A. (eds.) The Routledge Handbook of Second Language Acquisition. London: Routledge Taylor and Francis Group. p.p. 410-426

Flege, J. E. (1988). Factors affecting degree of perceived foreign accent in English sentences. The Journal of the Acoustical Society of America, vol. 84, núm. 1, p.p. 70-79.

Piske, T., Mackay, I. R., y Flege, J. E. (2001) Factors affecting degree of foreign accent in an L2: A review. Journal of Phonetics, vol.29, núm. 2, p.p. 191-215. Leech, G., Rayson, P., y Wilson, A. (2001). Word Frequencies in Written and Spoken English: based on the National British Corpus. UK: Routledge

Roach, P. (2009). English phonetics and phonology: $A$ practical course. Cambridge University Press.

Sanaphre, M. y Castellanos, M. J. (2018) "Utilizando la vista para pronunciar mejor" en Daniel Rodríguez V. y Joaquín A. Martínez M. (eds.) Exploración de principios y prácticas actuales en la enseñanza y aprendizaje de lenguas. México: Universidad Nacional Autónoma de México. p.p. 203-222

Toribio, A.J., Bullock, B.E., Botero, C.G. y Davis, K.A. (2005) Perservative phonetic effects in bilingual code-switching in Theoretical and Experimental Approaches to Romance Linguistics: Selected Papers from the 34th Linguistic Symposium on Romance Languages (LSRL), Salt Lake City, March 2004 (Vol. 272, p. 291). John Benjamins Publishing.
Weil, M. y Pullin, P. (2011) "English as a lingua franca in education. Internationalisation speaks English", Education permanente. Revue Suisse pour la formation continue, 2011-1, p.p. 28-29. 